\title{
Downregulation of the long non-coding RNA FOXD2-AS1 inhibits cell proliferation, migration and invasion in osteosarcoma
}

\author{
HAOMENG ZHANG $^{1 *}$, YAO LU $^{2 *}$, JUN WANG $^{3}$, TIAN ZHANG $^{4}$, CHUAN DONG $^{1}$, \\ XIAOXIANG LI ${ }^{1}$, XINLI WANG ${ }^{1}$, QIONG MA ${ }^{1}$, TONGTAO YANG ${ }^{1}$ and YONG ZHOU ${ }^{1}$ \\ ${ }^{1}$ Department of Orthopedic Oncology Institute, Tangdu Hospital, The Second Affiliated Hospital of Air Force \\ Medical University, Xi'an, Shaanxi 710038; ${ }^{2}$ Department of Orthopedic Surgery, Hong Hui Hospital, Xi'an Jiaotong \\ University College of Medicine, Xi'an, Shaanxi $710054 ;{ }^{3}$ Department of Neurosurgery, Xijing Institute of Clinical \\ Neuroscience, Xijing Hospital, The First Affiliated Hospital of Air Force Medical University, Xi'an, \\ Shaanxi 710032; ${ }^{4}$ Department of Pharmacy, Tangdu Hospital, The Second Affiliated Hospital of \\ Air Force Medical University, Xi'an, Shaanxi 710038, P.R. China
}

Received October 10, 2018; Accepted April 9, 2019

DOI: $10.3892 / \mathrm{mmr} .2019 .10254$

\begin{abstract}
Increasing amounts of long noncoding RNAs (lncRNAs) have been shown to be involved in the development of cancer. Recently, aberrant expression of the lncRNA forkhead box D2 adjacent opposite strand RNA1 (FOXD2-AS1) has been reported to be involved in the progression of several types of human cancer. However, the function and mechanism of FOXD2-AS1 in osteosarcoma (OS) are currently unclear. The present study aimed to investigate the function and mechanism of FOXD2-AS1 in OS. Firstly, it was revealed that the expression levels of FOXD2-AS1 were significantly upregulated in OS tissues and cells, compared with in adjacent tissues and normal cells, as determined using reverse transcription-quantitative polymerase chain reaction. Notably, the overall survival of patients with relatively high FOXD2-AS1 expression in OS tissues was significantly lower than that of patients with relatively low expression, as determined using Kaplan-Meier analysis. In addition, loss-of-function experiments were performed in vivo and in vitro to study the biological effects of FOXD2-AS1. The SOSP-9607 and U2OS OS cell lines were infected with lentivirus-mediated FOXD2-AS1 short hairpin RNA; subsequently, the alterations in cell phenotype and downstream molecules were evaluated.
\end{abstract}

Correspondence to: Professor Yong Zhou or Professor Tongtao Yang, Department of Orthopedic Oncology Institute, Tangdu Hospital, The Second Affiliated Hospital of Air Force Medical University, 569 Xinsi Road, Xi'an, Shaanxi 710038, P.R. China

E-mail: gukezy@163.com

E-mail: yangtt@fmmu.edu.cn

*Contributed equally

Key words: long non-coding RNA, osteosarcoma, proliferation, invasion, ribonucleotide reductase regulatory subunit $\mathrm{M} 2$
Knockdown of FOXD2-AS1 inhibited the proliferation, migration and invasion of OS cells. Furthermore, the number of cells in the $\mathrm{S}$ phase was significantly decreased, which was consistent with the results of the Cell Counting kit 8 proliferation assay. The expression levels of ribonucleotide reductase regulatory subunit M2 and phosphoglycerate dehydrogenase were decreased, as determined by western blotting, following FOXD2-AS1 knockdown. Finally, in a nude mouse model of tumorigenesis, it was revealed that, when FOXD2-AS1 expression was downregulated, tumor growth was significantly reduced and pulmonary metastatic nodules were markedly reduced. The results of the present study suggested that decreased FOXD2-AS1 expression may inhibit the growth, migration and invasion of tumor cells, and it may regulate downstream gene expression. In conclusion, these findings indicated that FOXD2-AS1 may be used as a potential therapeutic target and early tumor marker for the diagnosis and prognosis of OS.

\section{Introduction}

Osteosarcoma (OS) is a highly malignant primary bone neoplasm, which has a high rate in adolescents (1). In the majority of patients with OS, survival is significantly prolonged by radiotherapy and chemotherapy combined with surgery; however, patients with distant metastasis have poor treatment options, particularly when it has spread to the lungs (2). With the advent of high-throughput sequencing technology, interest in long non-coding RNAs (lncRNAs) is gradually increasing. IncRNAs are a class of RNA molecules that are $>200 \mathrm{nt}$ long and lack an open reading frame $(3,4)$. Numerous studies have revealed that lncRNAs are involved in biological developmental regulatory processes, including cell differentiation, growth and apoptosis $(5,6)$. Furthermore, several lncRNAs have been demonstrated to be involved in various human diseases (7). Notably, aberrantly expressed lncRNAs have been reported to serve roles in inhibiting and promoting cancer growth in different tumor types (8). 
Recently, several studies have confirmed the dysregulation of lncRNA expression in OS, which is associated with the progression, metastasis and prognosis of the disease $(2,9)$. In particular, the dysregulated lncRNAs identified included lncRNA-activated by Transforming Growth Factor $\beta$ and lncRNA-taurine upregulated gene 1 . Therefore, lncRNAs may serve as diagnostic markers for OS. A novel oncogenic lncRNA, forkhead box D2 adjacent opposite strand RNA1 (FOXD2-AS1), has been shown to serve an important role in various tumor types, including liver, bladder and non-small cell lung cancer. For example, high expression of FOXD2-AS1 regulates the proliferation and metastasis of non-small cell lung cancer cells via the Wnt/ $\beta$-catenin signaling pathway; downregulation of FOXD2-AS1 inhibits the proliferation, metastasis and invasion of hepatocellular carcinoma cells; and FOXD2-AS1 promotes bladder cancer cell proliferation, migration and invasion by regulating a feedback loop with Akt and E2F transcription factor 1 (E2F1) (10-12). However, to the best of our knowledge, the biological function and potential molecular mechanisms of FOXD2-AS1 in human OS are currently unclear.

The present study aimed to investigate the function and mechanism of FOXD2-AS1 in OS. In the present study, FOXD2-AS1 expression was significantly increased in OS tissues and cell lines. In addition, Kaplan-Meier analysis revealed that FOXD2-AS1 expression was closely related to the survival of patients with OS, thus suggesting that FOXD2-AS1 may be a novel biomarker for the effective diagnosis of OS. Knockdown of FOXD2-AS1 inhibited the proliferation of OS cells, and suppressed their migratory and invasive abilities. In conclusion, the present results revealed that FOXD2-AS1 may be an effective target for the treatment of patients with OS.

\section{Materials and methods}

Clinical specimens. In the present study, 40 patients with OS (age range, 6-51; 24 male patients and 16 female patients) were recruited in the Department of Orthopedic Oncology Institute, Tangdu Hospital, The Second Affiliated Hospital of Air Force Medical. The patients underwent resection and the specimens were collected; the samples were collected between July 2012 and August 2014 at The Second Affiliated Hospital of Air Force Medical University. None of the patients underwent chemotherapy or radiotherapy prior to surgery. The collected specimens were obtained with the written informed consent of the patients or their families. Informed consent was obtained from the parents of patients $<16$ years old. The collected OS tissues and adjacent tissue controls $(>3 \mathrm{~cm}$ from the edge of the tumor) were immediately stored in liquid nitrogen for subsequent use. The use of human OS tissue was approved by the Air Force Medical University Ethics Committee.

Relative expression levels of FOXD2-AS1 were analyzed by reverse transcription-quantitative polymerase chain reaction (RT-qPCR). Data are presented as $\log _{2}$ fold change $\left(2^{-\Delta \Delta C q}\right)$. The samples were divided into two groups (high and low) based on the median expression level of FOXD2-AS1. Subsequently, the two groups were examined using the Kaplan-Meier analysis followed by log-rank test.

Cell culture. The human OS cell line SOSP-9607 was established by our laboratory and maintained in our laboratory (13).
Other cell lines used in this study, hFOB1.19, U2OS, MG63 and SAOS2, were purchased from the American Type Culture Collection. SOSP-9607 cell line was cultured in RPMI-1640 medium (HyClone; GE Healthcare Life Sciences) containing $10 \%$ fetal bovine serum (FBS; HyClone; GE Healthcare Life Sciences) at $37^{\circ} \mathrm{C}$ in a humidified atmosphere with $5 \% \mathrm{CO}_{2}$. U2OS, SAOS2 and MG63 cells were cultured in DMEM (HyClone; GE Healthcare Life Sciences) containing 10\% FBS at $37^{\circ} \mathrm{C}$ in a humidified atmosphere with $5 \% \mathrm{CO}_{2}$. The hFOB1.19 cell line was grown in DMEM containing $10 \%$ FBS and $0.3 \mathrm{mg} / \mathrm{ml} \mathrm{G} 418$ (Sigma-Aldrich; Merck KGaA) at $33.5^{\circ} \mathrm{C}$ in a humidified atmosphere containing $5 \% \mathrm{CO}_{2}$.

Construction of lentiviral vectors containing green fluorescent protein (GFP) and infection. To stably silence FOXD2-AS1, a lentiviral vector (Hanbio Biotechnology Co., Ltd.) that specifically and stably expressed a FOXD2-AS1 short hairpin RNA (shRNA). The FOXD2-AS1 shRNA was constructed by Hanbio Biotechnology Co., Ltd. The FOXD2-AS1 shRNA interference sequence was 5'-GATCCGCGAAGAGTACGTTGCTATTTC AAGAGAATAGCAACGTACTCTTCGCTTTTTTC-3', the shRNA control sequence (scramble) was 5'-TTCTCCGAACGT GTCACGTAA-3'. SOSP-9607 and U2OS cells (2x104 cells/well) were infected with the virus at a multiplicity of infection of 100 and 50, respectively. After overnight incubation at $37^{\circ} \mathrm{C}$, the virus-containing culture supernatant was removed and replaced with fresh virus-free medium. After $48 \mathrm{~h}$ of culture, the infection efficiency was determined by observing the intensity of GFP expression in the cells under an Olympus IX71 fluorescence microscope (Olympus Corporation). Cells expressing GFP were selected for further analysis. After infection, cells were cultured for $96 \mathrm{~h}$ prior to subsequent experiments.

$R T$ - $q P C R$. Total RNA was extracted from all OS tissues and cells using TRIzol ${ }^{\circledR}$ reagent (Invitrogen; Thermo Fisher Scientific, Inc.) according to the manufacturer's protocol. Total RNA was dissolved in RNase-free water and the concentration was measured using an Epoch spectrophotometer (ND-1000 spectrophotometer; NanoDrop; Thermo Fisher Scientific, Inc.). cDNA was synthesized using the 5X-All-In-One RT MasterMix kit (Applied Biological Materials, Inc.) according to the manufacturer's protocol, with a Bio-Rad MyCycler system (Bio-Rad Laboratories, Inc.), with the following conditions: $37^{\circ} \mathrm{C}$ for $15 \mathrm{~min}$ and $85^{\circ} \mathrm{C}$ for $5 \mathrm{sec}$, followed by cooling to $4^{\circ} \mathrm{C}$. The synthesized cDNA was then subjected to qPCR using the EvaGreen 2X qPCR MasterMix kit (Applied Biological Materials, Inc.) on the Rotor-Gene Q 2plex system (Qiagen $\mathrm{GmbH}$ ). qPCR thermocycling conditions were as follows: Denaturation at $95^{\circ} \mathrm{C}$ for $10 \mathrm{~min}$, followed by 40 cycles at $95^{\circ} \mathrm{C}$ for $15 \mathrm{sec}, 65^{\circ} \mathrm{C}$ for $10 \mathrm{sec}$ and $72^{\circ} \mathrm{C}$ for $15 \mathrm{sec}$. The standard curves were calculated and the relative quantification of gene expression was assessed. GAPDH expression was used as a standardized internal reference and the $2^{-\Delta \Delta \mathrm{Cq}}$ method was used for relative quantification (14). The sequences of all primers are presented in Table I.

Cell Counting kit-8 (CCK8) assay. SOSP-9607 and U2OS cells were seeded into 96 -well tissue culture plates $\left(3 \times 10^{3}\right.$ cells/well). The CCK8 assay was performed at 24,48, 72 and $96 \mathrm{~h}$. Following the addition of $10 \mu \mathrm{l} /$ well CCK8 reagent (Dojindo Molecular 
Table I. Sequences of primers and probes.

\begin{tabular}{ll}
\hline Primer name & \multicolumn{1}{c}{ Sequence } \\
\hline GAPDH & \\
Forward & 5'CGCTCTCTGCTCCTCCTGTTC3' \\
Reverse & 5'CCGTTGACTCCGACCTTCAC3' \\
FOXD2-AS1 & \\
Forward & 5'AGGGACAGCCAAGAATACTC3' \\
Reverse & 5'GGGACTCAGAAGGGTTACAC3' \\
RRM2 & \\
Forward & 5'CCACGGAGCCGAAAACTAAG3' \\
Reverse & 5'CTCTGCCTTCTTATACATCTGCC3' \\
PHGDH & \\
Forward & 5'ATCTGCGGAAAGTGCTCATC3' \\
Reverse & 5'GCAGAGCGAACAATAAGGC3' \\
COL5A1 & \\
Forward & 5'TACCCTGCGTCTGCATTTCC3' \\
Reverse & 5'GCTCGTTGTAGATGGAGACCA3' \\
FZD1 & \\
Forward & 5'ATCTTCTTGTCCGGCTGTTACA3' \\
Reverse & 5'GTCCTCGGCGAACTTGTCATT3' \\
EFNA4 & \\
Forward & 5'CCCCCTCTGTCTCTTGCTATT3' \\
Reverse & 5'TCTTGTCGGTCTGAATTGGCA3' \\
EPHB2 & \\
Forward & 5'GTGTGCAACGTGTTTGAGTCA3' \\
Reverse & 5'ACGCACCGAAAACTTCATCTC3' \\
FOXD2-AS1 probe & 5'-DIG-CGACCCGGACGCCACTGA \\
& TAGCAAC-DIG-3' \\
\hline COL5A1, & \\
\hline
\end{tabular}

COL5A1, collagen type $\mathrm{V} \alpha 1$ chain; EFNA4, ephrin A4; EPHB2, $\mathrm{EPH}$ receptor $\mathrm{B} 2$; FOXD2-AS1, forkhead box D2 adjacent opposite strand RNA1; FZD1, frizzled class receptor 1; PHGDH, phosphoglycerate dehydrogenase; RRM2, ribonucleotide reductase regulatory subunit M2.

Technologies, Inc.), cells were incubated for $2 \mathrm{~h}$ at $37^{\circ} \mathrm{C}$ and absorbance was measured at $450 \mathrm{~nm}$ using an Infinite M200 Pro Multifunctional microplate reader (Tecan Group Ltd).

Colony formation assay. SOSP-9607 and U2OS cells infected with the FOXD2-AS1 shRNA were seeded into 6-well plates (500 cells/well) and cultured at $37^{\circ} \mathrm{C}$ in a humidified atmosphere containing $5 \% \mathrm{CO}_{2}$. The culture medium was changed every 2 days. After 2 weeks, the macroscopic observation of cell growth was terminated. The cells were washed twice with PBS, fixed in methanol for 40 min, stained with $1 \%$ crystal violet dye for $5 \mathrm{~min}$ at room temperature and rinsed twice with water. Finally, images were captured to count the colonies.

5-Ethynyl-2-deoxyuridine (EdU) assay. The EdU experiment was performed using the iClick ${ }^{\mathrm{TM}}$ EDU Andy Fluor $^{\mathrm{TM}} 647$ Imaging kit (cat. no. A006; GeneCopoeia, Inc.) according to the manufacturer's protocol. OS cells were treated with $10 \mu \mathrm{m}$
EdU solution and were incubated for $2 \mathrm{~h}$ at $37^{\circ} \mathrm{C}$. Next, $200 \mu \mathrm{l}$ 1X Hoechst $33342(5 \mu \mathrm{g} / \mathrm{ml})$ was added to each well and incubated at room temperature for $15 \mathrm{~min}$ in the dark. Images were captured under an Olympus IX71 fluorescence microscope (Olympus Corporation). Andy Fluor 647 azide was detected at $650 \mathrm{~nm}$. Hoechst 33342 was detected at $350 \mathrm{~nm}$.

Cell migration and invasion assays. Briefly, 24-well Transwell plates (pore size, $8 \mu \mathrm{m}$; Corning, Inc.) were used for cell invasion and migration assays. For the cell migration assay, $5 \times 10^{4}$ OS cells were seeded into the upper chambers of 24-well plates in $200 \mu \mathrm{l}$ serum-free DMEM. The lower chamber contained DMEM supplemented with $10 \%$ FBS. After $20 \mathrm{~h}$ at $37^{\circ} \mathrm{C}$, the non-migrating cells were gently removed from the upper side of the chamber with a cotton swab, and the migrated cells were washed twice with PBS, fixed with $95 \%$ alcohol for $10 \mathrm{~min}$ and stained with $1 \%$ crystal violet for $5 \mathrm{~min}$. Finally, images were captured and cells were counted under an light inverted microscope (Olympus Corporation).

For the invasion assay, the wells were pretreated with Matrigel (BD Biosciences), and OS cells $\left(1 \times 10^{5}\right.$ cells per chamber) were seeded into the upper chamber. The lower chamber contained DMEM supplemented with $10 \%$ FBS. The subsequent steps were the same as those conducted for the cell migration assay.

Flow cytometry. To analyze cell cycle distribution, a sufficient number $\left(1 \times 10^{6}\right)$ of stably infected cells was collected, fixed overnight at $4^{\circ} \mathrm{C}$ in $500 \mu 170 \%$ cold ethanol and washed three times with PBS. Subsequently, the cells were incubated with $450 \mu \mathrm{l}$ propidium iodide (PI) and $50 \mu \mathrm{l}$ RNase A using the Annexin V-APC/PI apoptosis detection Kit (Nanjing KeyGen Biotech, Co., Ltd.) according to the manufacturer's protocol in the dark at room temperature for $1 \mathrm{~h}$. Cell cycle progression was quantified using a NovoCyte 2040R flow cytometer (ACEA Biosciences, Inc.), the data were analyzed using the NovoExpress Software (version1.4; ACEA Biosciences, Inc.).

Nuclear fractionation analysis and fluorescence in situ hybridization (FISH). Nuclear and cytoplasmic FOXD2-AS1 was isolated from $1 \times 10^{7}$ SOSP-9607 and U2OS cells using the PARISTM kit (Ambion; Thermo Fisher Scientific, Inc.) according to the manufacturer's protocol. SOSP-9607 and U2OS cells were used for RNA-FISH analysis. The relative localization of the transcript was calculated according to the manufacturer's protocol (PARIS ${ }^{\mathrm{TM}}$ kit; Ambion; Thermo Fisher Scientific, Inc.). The cell suspension $\left(1 \times 10^{5} \mathrm{cells} / \mathrm{ml}\right)$ was pipetted onto slides, fixed in $4 \%$ paraformaldehyde for $20 \mathrm{~min}$ at room temperature and washed with PBS (pH 7.4) three times ( $5 \mathrm{~min} / \mathrm{wash}$ ). Following digestion with proteinase $\mathrm{K}$ $(20 \mu \mathrm{g} / \mathrm{ml})$ for $5 \mathrm{~min}$ at $37^{\circ} \mathrm{C}$, the samples were washed three times in PBS (5 min/wash). Subsequently, hybridization with the digoxigenin (DIG)-labeled FOXD2-AS1_lnc probe (8 ng/ $\mu$ l; Wuhan Servicebio Technology Co., Ltd.) was carried out at $37^{\circ} \mathrm{C}$ overnight. The sequence of the probe is presented in Table I. After hybridization, the slides were washed with $2 \mathrm{X}$ SSC for $10 \mathrm{~min}$ at $37^{\circ} \mathrm{C}, 1 \mathrm{X} \mathrm{SSC}$ two times for $5 \mathrm{~min}$ at $37^{\circ} \mathrm{C}$, and $0.5 \mathrm{X} \mathrm{SSC}$ for $10 \mathrm{~min}$ at $37^{\circ} \mathrm{C}$. Subsequently, the blocking buffer containing 5\% BSA (Wuhan Servicebio Technology Co., Ltd.) was added and cells were incubated for $30 \mathrm{~min}$ at 
room temperature. The slides were then incubated with the anti-DIG-488 antibody (1:300; Jackson ImmunoResearch Laboratories, Inc.; cat. no. 200-482-156) at $37^{\circ} \mathrm{C}$ for $50 \mathrm{~min}$ and washed three times in PBS (5 min/wash). DAPI solution was then added dropwise and the cells were incubated in the dark for $8 \mathrm{~min}$ at room temperature. After washing, anti-fade mounting medium (cat. no. G1401; Wuhan Servicebio Technology Co., Ltd.) was pipetted onto the slides. The images were acquired using a Nikon direct fluorescence microscope (Nikon Corporation).

Western blotting. Total cellular protein was extracted using RIPA buffer (Beijing ComWin Biotech Co., Ltd.) supplemented with $1 \%$ protease inhibitor (100X; Beijing ComWin Biotech Co., Ltd.), and protein concentration was quantified with a BCA protein assay kit. Proteins (20-30 $\mu \mathrm{g})$ were separated by SDS-PAGE, using 6-10\% acrylamide gels, and were then transferred to a PVDF Immobilon-P membrane (EMD Millipore). After blocking for $2 \mathrm{~h}$ at $37^{\circ} \mathrm{C}$ with $5 \%$ skim milk, membranes were incubated overnight with a primary antibody anti-phosphoglycerate dehydrogenase (PHGDH; cat. no. BS71734; 1:1,000; Bioworld Technology, Inc.) and ribonucleotide reductase regulatory subunit M2 (RRM2; cat. no. BS7520; 1:1,000; Bioworld Technology, Inc.) at $4^{\circ} \mathrm{C}$, followed by incubation with a horseradish peroxidase-labeled goat anti-rabbit immunoglobulin $\mathrm{G}$ secondary antibody (cat. no. BS10043; 1:2,000; Bioworld Technology, Inc.) for $2 \mathrm{~h}$ at $37^{\circ} \mathrm{C}$. The ECL detection reagent (EMD Millipore) was used to visualize the bands using an ECL detection system (Thermo Fisher Scientific, Inc.).

Tumor xenograft assay. Nude BALB/c female mice (age, 4 weeks; weight, 16-22 g) were purchased from the experimental animal center of the Air Force Medical University, and were bred under the following pathogen-free conditions: 12-h light/dark cycle; temperature, $24-28^{\circ} \mathrm{C}$; humidity, $40-60 \%$. Mice had free access to food and water. The animal feed and heating pad were purchased from the experimental animal center of the Air Force Medical University, and were autoclaved and vacuum packed. A total of eight nude mice took part in the experimental study.

For the tumorigenicity experiment, SOSP-9607 cells infected with FOXD2-AS1 shRNA or scramble shRNA were suspended in PBS, and the cell concentration was adjusted to $1 \times 10^{8} / \mathrm{ml}$. Each female BALB/c nude mouse was injected with a $30-\mu 1$ cell suspension ( $n=4$ mice/group) into the tibial plateau, and tumor size was measured every 6 days. After 36 days, mice were sacrificed by cervical dislocation. The tumor and lung tissues of each group were removed and fixed for $2 \mathrm{~h}$ at $4^{\circ} \mathrm{C}$ in $4 \%$ paraformaldehyde for subsequent pathological and immunohistochemical examination, to detect the protein expression level of Ki-67. The study was approved by the Animal Care and Use Committee of the Second Affiliated Hospital of the Air Force Medical University.

Immunohistochemistry. The tumor tissue was fixed in $4 \%$ paraformaldehyde at $4^{\circ} \mathrm{C}$ for $2 \mathrm{~h}$, dehydrated in alcohol and embedded in paraffin. The sections were incubated in $3 \%$ hydrogen peroxide at room temperature for $25 \mathrm{~min}$ in the dark. Subsequently, the sections were washed with PBS ( $\mathrm{pH} 7.4$ ).
Then, the samples were incubated with 3\% BSA (cat. no. G5001; Wuhan Servicebio Technology Co., Ltd.) and blocked at room temperature for $30 \mathrm{~min}$. After removing the blocking buffer, the sections were incubated overnight at $4^{\circ} \mathrm{C}$ with a primary antibody anti-Ki-67 Rabbit mAb (1:500 cat. no. GB13030-2; Wuhan Servicebio Technology Co., Ltd.). The slices were incubated in PBS (pH 7.4) and washed three times with PBS. Next, the sections were incubated with a secondary antibody goat anti-rabbit immunoglobulin G (1:1,000; cat. no. BS10043; Bioworld Technology, Inc.) for $50 \mathrm{~min}$ at room temperature. Immunohistochemical staining results were observed using the Olympus BX51 light microscope (Olympus Corporation; magnification, $\mathrm{x} 400$ ).

Statistical analysis. All data were statistically analyzed using SPSS 21.0 software (IBM Corp.). All experiments were repeated at least three times and the data are expressed as the means \pm standard deviation. Comparisons of data between two groups were performed using a two-tailed Student's t-test comparisons between multiple groups were performed using one-way analysis of variance with Fisher's least significance difference post hoc test. $\mathrm{P}<0.05$ was considered to indicate a statistically significant difference.

\section{Results}

FOXD2-AS1 is upregulated in OS tissues and cells. In order to investigate the biological function of FOXD2-AS1 in OS, the expression levels of FOXD2-AS1 were detected in OS tissues and cells by RT-qPCR. As shown in Fig. 1, the expression levels of FOXD2-AS1 were detected in 40 samples of OS and adjacent tissues. The results revealed that FOXD2-AS1 expression was upregulated in $80 \%$ of OS tissue samples $(32 / 40)$ compared with in adjacent tissues (Fig. 1A). Furthermore, the high expression of FOXD2-AS1 was associated with a significantly lower survival time compared with relatively low FOXD2-AS1 expression (Fig. 1B). In addition, RT-qPCR demonstrated that the expression levels of FOXD2-AS1 were increased in OS cells (9607, U2OS, MG63 and SAOS2) compared with in hFOB1.19 cells (Fig. 1E).

Knockdown of FOXD2-AS1 inhibits OS cell proliferation by inducing cell cycle arrest. To investigate the role of FOXD2-AS1 in the proliferation of OS cells, lentivirus-mediated FOXD2-AS1 shRNA infection was conducted in OS cell lines (SOSP-9607 and U2OS), in order to establish SOSP-9607 and U2OS cell lines with stable low expression of FOXD2-AS1. The knockdown efficiency of FOXD2-AS1 was verified by RT-qPCR after $72 \mathrm{~h}$ of infection (Fig. 2A). The results of a CCK8 assay revealed that downregulation of FOXD2-AS1 inhibited the proliferation of OS cells (Fig. 2B). In addition, FOXD2-AS1 knockdown reduced the colony-forming ability of OS cells (Fig. 2C and D). The EdU assay further confirmed that knockdown of FOXD2-AS1 inhibited cell proliferation (Fig. 2E). Finally, flow cytometry demonstrated that downregulation of FOXD2-AS1 significantly reduced the abundance of cells in $\mathrm{S}$ phase of the cell cycle (Fig. 3C).

Decreased expression of FOXD2-AS1 inhibits the migration and invasion of $O S$ cells. Specific invasion and migration assays 

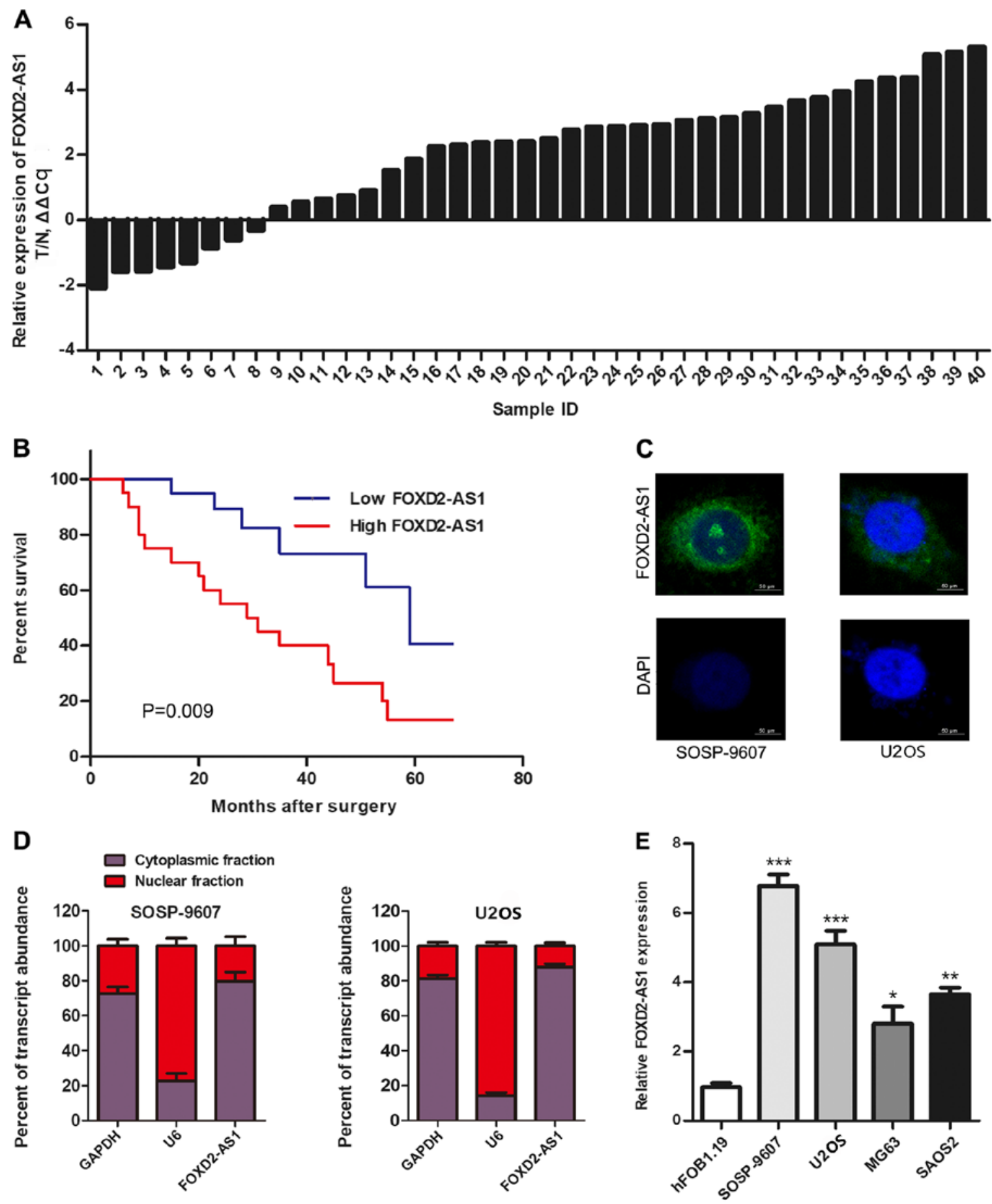

Figure 1. Expression of FOXD2-AS1 in OS cell lines and tissues. (A) Relative expression levels of FOXD2-AS1 in 40 paired specimens of OS and adjacent tissues, as quantified by RT-qPCR. Data are presented as $\log 2$ fold change. (B) Association between the expression levels of FOXD2-AS1 and survival for patients with OS, as determined by Kaplan-Meier analysis. (C) Determination of subcellular localization of FOXD2-AS1 in OS cells by fluorescence in situ hybridization. (D) Expression of FOXD2-AS1 in the cytoplasm and nuclei of OS cells; U1 and GAPDH were used as controls. (E) Expression levels of FOXD2-AS1 in OS cell lines (9607, U2OS, MG63 and SAOS2) and normal osteoblasts (hFOB1.19), as quantified by RT-qPCR. "P<0.05, ${ }^{* *} \mathrm{P}<0.01$ and ${ }^{* *} \mathrm{P}<0.01$ vs. hFOB1.19. Data are expressed as the means \pm standard deviation. FOXD2-AS1, forkhead box D2 adjacent opposite strand RNA1; N, normal; OS, osteosarcoma; RT-qPCR, reverse transcription-quantitative polymerase chain reaction; T, tumor.

were used to investigate the association between FOXD2-AS1 and the migratory/invasive abilities of OS cells. The results confirmed that downregulation of FOXD2-AS1 inhibited SOSP-9607 and U2OS tumor cell migration and invasion (Fig. 3A and B). These results revealed that FOXD2-AS1 may act as a tumor promoter by enhancing cell invasion and migration.
Localization of FOXD2-AS1 in cells and its potential downstream genes. To investigate the mechanism by which FOXD2-AS1 exerts its effects on OS cells, this study revealed that FOXD2-AS1 expression was predominantly abundant in the cytoplasm using FISH and RNA isolation experiments (Fig. 1C and D). lncRNAs located in the cytoplasm can act as competing endogenous RNAs (ceRNAs). Therefore, 
A

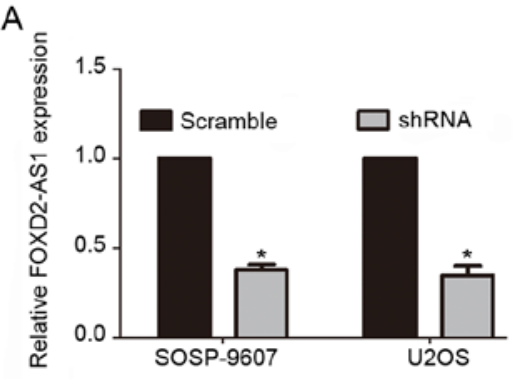

C
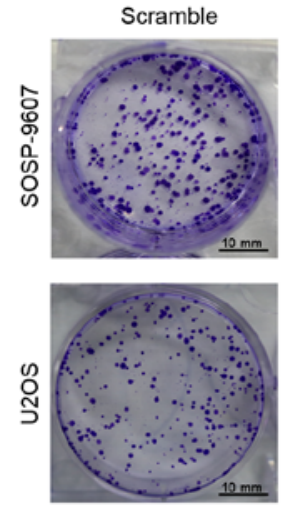

$E$
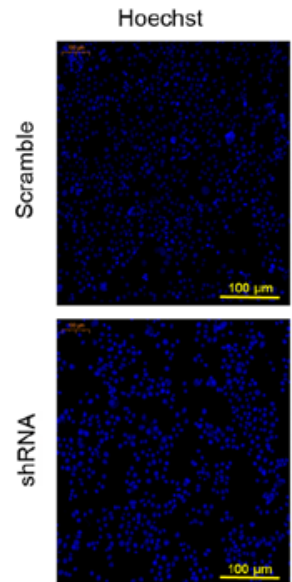

B
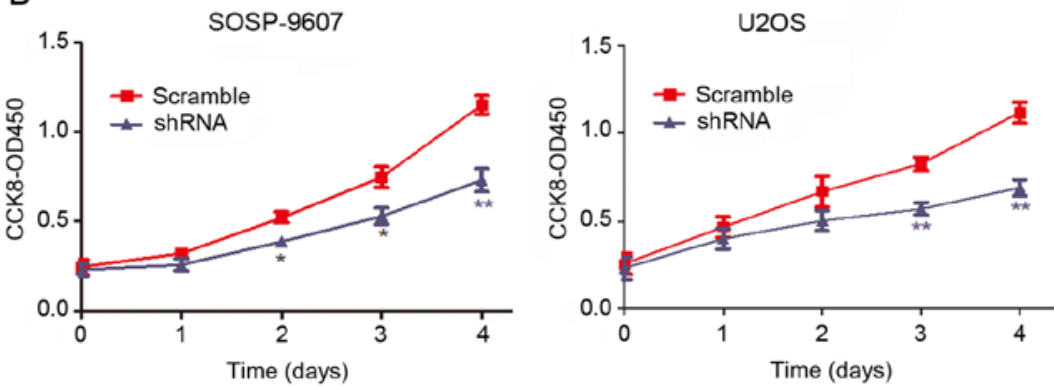

ShRNA
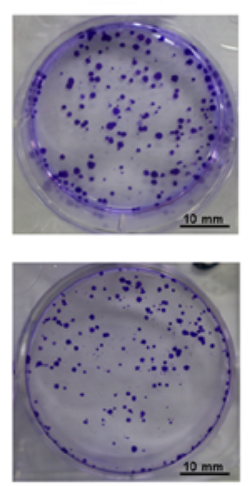

Edu
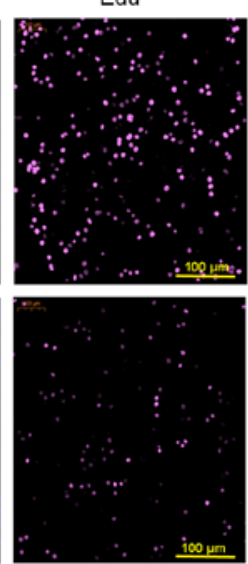

SOSP-9607
D
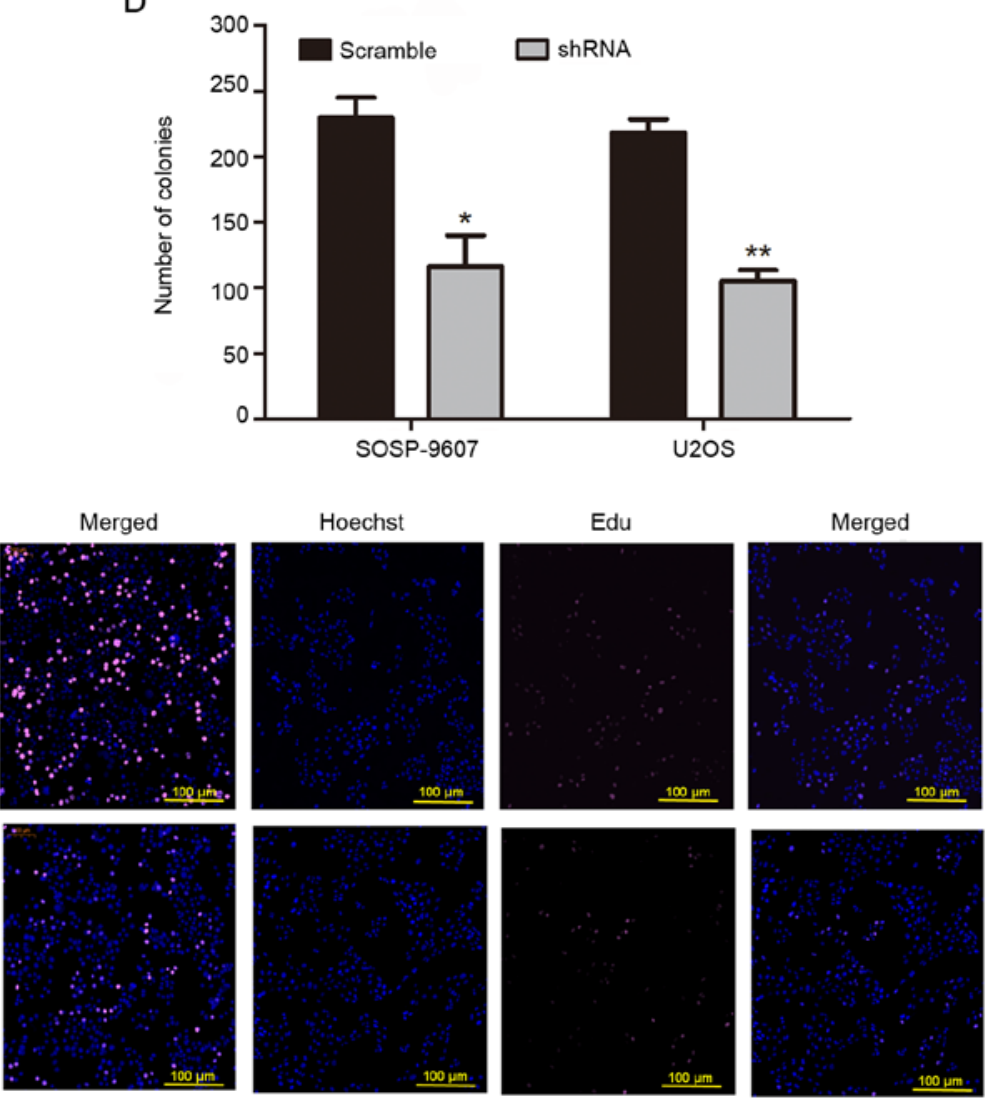

U2OS

Figure 2. FOXD2-AS1 promotes the growth of OS cells in vitro. (A) Expression levels of FOXD2-AS1 in SOSP-9607 and U2OS OS cells post-infection with FOXD2-AS1 shRNA or scramble shRNA. (B) CCK8 assay revealed that FOXD2-AS1 knockdown inhibited the proliferation of OS cells. (C and D) Colony formation assays demonstrated that stable low expression of FOXD2-AS1 significantly reduced colony formation compared with the scramble group. (E) EdU assays further confirmed that knockdown of FOXD2-AS1 reduced the proliferation of OS cells. ${ }^{*} \mathrm{P}<0.05,{ }^{* *} \mathrm{P}<0.01 \mathrm{vs}$. scramble shRNA. Data are presented as the means \pm standard deviation. CCK8, Cell Counting kit 8; EdU, 5-ethynyl-2-deoxyuridine; FOXD2-AS1, forkhead box D2 adjacent opposite strand RNA1; OD, optical density; OS, osteosarcoma; shRNA, short hairpin RNA.

FOXD2-AS1 may act as a ceRNA to sponge microRNAs and thus regulate mRNA expression. To determine the downstream genes of FOXD2-AS1 in OS cells, transcriptomes of five pairs of OS tissues and adjacent tissues were sequenced (data not shown); the results revealed that, when FOXD2-AS1 was upregulated, the mRNA expression levels of ephrin A4, collagen type $\mathrm{V} \alpha 1$ chain, EPH receptor B2, frizzled class receptor 1, PHGDH and RRM2 may be upregulated (data not shown). Therefore, it was hypothesized that FOXD2-AS1 may regulate the expression of these six mRNAs. To confirm this hypothesis, FOXD2-AS1 was knocked down and the expression of these six mRNAs was simultaneously detected. The results of RT-qPCR demonstrated that the downregulation of
FOXD2-AS1 significantly downregulated the expression levels of PHGDH and RRM2 (Fig. 4A). Western blotting further confirmed that the expression levels of PHGDH and RRM2 were reduced compared with in the scramble group (Fig. 4B).

FOXD2-AS1 knockdown inhibits the growth of SOSP-9607 OS cell line in vivo. To further confirm the effects of FOXD2-AS1 on the proliferation of SOSP-9607 OS cells in vivo, two groups of stable cells (scramble shRNA- and FOXD2-AS1 shRNA-infected SOSP-9607 cells) were injected into the tibial plateau of nude mice. To evaluate tumor growth, the volume of the in situ tumor was measured every 6 days post-inoculation. The following formula was used to measure tumor volume: 
A

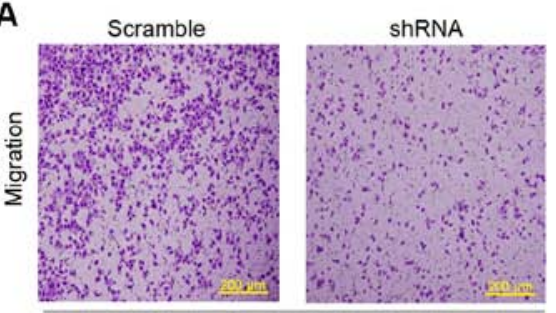

SOSP-9607

B

Scramble

shRNA
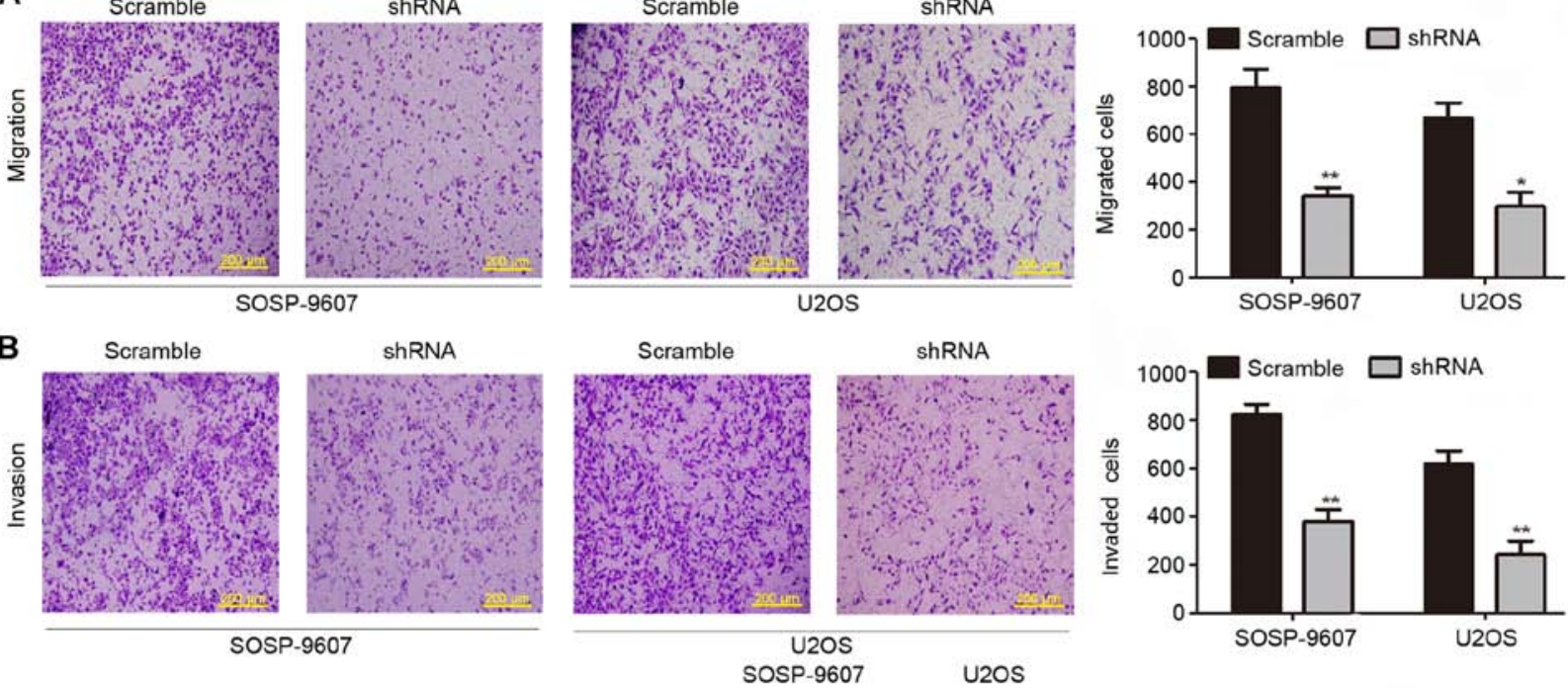

C
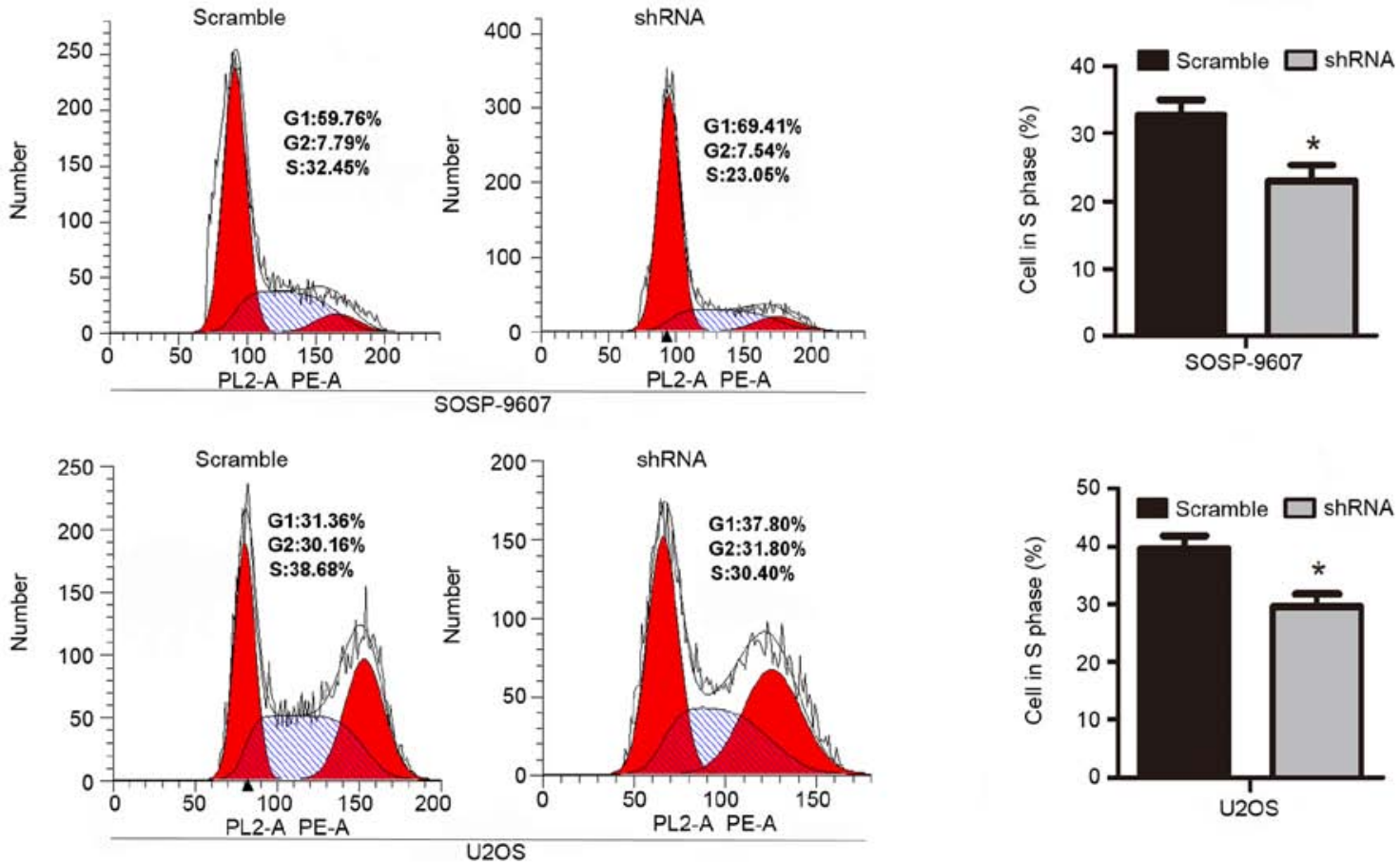

Figure 3. Effect of FOXD2-AS1 knockdown on the biological behavior of OS cells. (A and B) Cell migration and invasion assays demonstrated that knockdown of FOXD2-AS1 inhibited the migration and invasion of SOSP-9607 and U2OS OS cells. (C) Cell cycle analysis demonstrated that high FOXD2-AS1 expression increased the proportion of S-phase cells in OS cultures. ${ }^{*} \mathrm{P}<0.05,{ }^{* *} \mathrm{P}<0.01$ vs. scramble shRNA. Data are presented as the means \pm standard deviation. FOXD2-AS1, forkhead box D2 adjacent opposite strand RNA1; OS, osteosarcoma; shRNA, short hairpin RNA.

$1 / 2 \times \mathrm{L}^{2} \mathrm{~W}^{2}$, where $\mathrm{L}$ refers to length and $\mathrm{W}$ to width. The growth curves revealed that FOXD2-AS1 knockdown significantly slowed the growth of OS (Fig. 5A-C) and reduced its propensity for lung metastasis (Fig. 5D). Histopathological evaluation of orthotopic tumors and lung metastases in nude mice was performed to compare the shRNA group and the scramble group (Fig. 5E-G). The present results suggested that downregulation of FOXD2-AS1 expression inhibited tumor growth and lung metastasis in vivo.

\section{Discussion}

OS is a primary malignant tumor that commonly occurs in children and adolescents (1), which is characterized by high lung metastatic potential and a poor prognosis. In recent years, with the development of neoadjuvant chemotherapy and combination therapy, the survival rate of patients with early metastasis-free OS has increased by 55 to $70 \%(15,16)$. Nevertheless, it is considered that developments surrounding the survival rate and the therapeutic measures available for OS have entered a relatively stable period. Notably, $15-20 \%$ of patients with OS present with metastases at the time of diagnosis, which are mostly located in the lungs (17). Therefore, it is necessary to explore novel clinical targets for the treatment of patients with OS, and to further elucidate the pathogenesis of OS.

In recent years, numerous studies have demonstrated that abnormal expression of lncRNAs is closely associated with the 
A

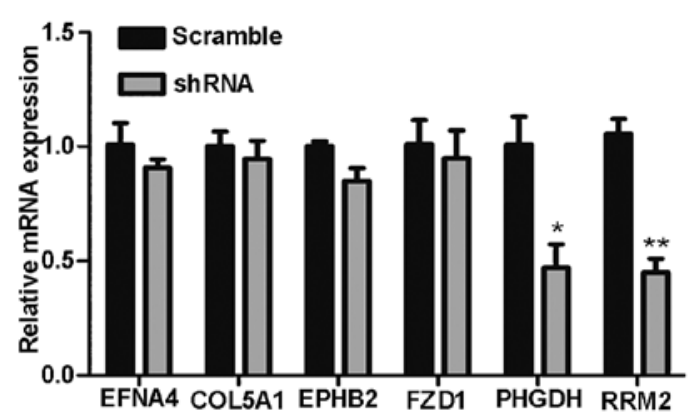

U2OS

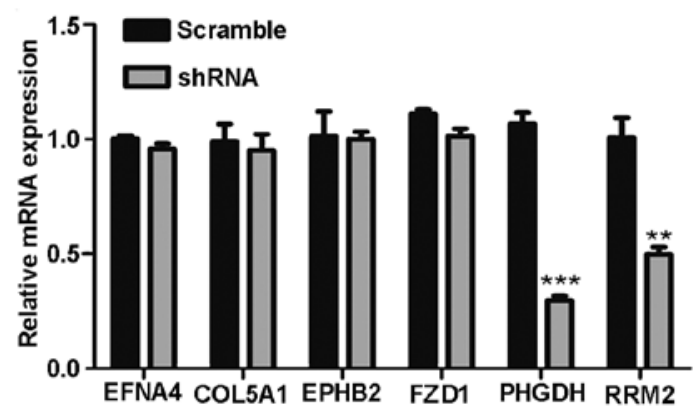

B

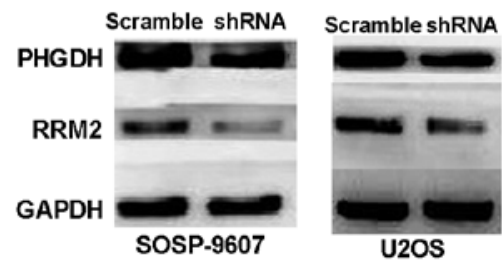

Figure 4. FOXD2-AS1 affects the expression of downstream genes and proteins. (A) mRNA expression levels of EFNA4, COL5A1, EPHB2, FZD1, PHGDH and RRM2 in SOSP-9607 and U2OS osteosarcoma cells post-infection with FOXD2-AS1 shRNA or scramble shRNA. (B) Further verification by western blotting revealed that FOXDA2-AS1 knockdown decreased the expression levels of the downstream proteins PHGDH and RRM2. "P $<0.05$, ${ }^{* *} \mathrm{P}<0.01$ and ${ }^{* * *} \mathrm{P}<0.001$ vs. scramble shRNA. Data are presented as the means \pm standard deviation. COL5A1, collagen type V $\alpha 1$ chain; EFNA4, ephrin A4; EPHB2, EPH receptor B2; FOXD2-AS1, forkhead box D2 adjacent opposite strand RNA1; FZD1, frizzled class receptor 1; shRNA, short hairpin RNA; PHGDH, phosphoglycerate dehydrogenase; RRM2, ribonucleotide reductase regulatory subunit M2.

occurrence and development of cancer $(18,19)$. Furthermore, aberrantly expressed lncRNAs can promote the growth and metastasis of tumors; they can act as tumor suppressor genes and oncogenes (20-22). For example, the lncRNA urothelial cancer-associated 1 has an oncogenic role in breast cancer and lung cancer, whereas the lncRNA TCF21 antisense RNA inducing promoter demethylation suppresses genes through methylation to prevent the occurrence of cancer (20-22). Accordingly, the abnormal expression of IncRNAs in OS may be closely related to its biological behavior. For example, it has been established that lncRNAs are closely related to the proliferation, metastasis, apoptosis and poor prognosis of OS $(23,24)$.

Recently, it has been reported that aberrant expression of FOXD2-AS1 serves a crucial role in carcinogenesis. A previous study revealed that FOXD2-AS1 mediates chondrocyte proliferation in osteoarthritis by regulating the expression of cyclin D1 via sponging microRNA-206 (25). Another study demonstrated that FOXD2-AS1 promotes bladder cancer cell proliferation, migration and invasion by regulating a feedback loop with Akt and E2F1 (10). Yang et al (26)revealed that FOXD2-AS1 promotes colorectal cancer cell proliferation, and that epithelial-mesenchymal transition and Notch signaling pathway activity are decreased in cells following knockdown of FOXD2-AS1. However, few studies have investigated the biological role of FOXD2-AS1 in OS.

To elucidate the role of FOXD2-AS1 in the progression of OS, FOXD2-AS1 expression was quantified in 40 pairs of OS tissues and adjacent tissues using RT-qPCR. To further confirm the role of FOXD2-AS1 in OS, the expression of FOXD2-AS1 was validated by RT-qPCR in OS cell lines (SOSP-9607, U2OS, SAOS2 and MG63) and normal osteoblasts (hFOB1.19).
The results demonstrated that FOXD2-AS1 expression was increased in OS tissues and cells compared with in adjacent tissues and normal osteoblasts. Knockdown of FOXD2-AS1 expression inhibited OS cell proliferation, migration and invasion. In addition, dysregulation of cell cycle transitions is an important marker of cell carcinogenesis (27). Therefore, to investigate the underlying mechanism of FOXD2-AS1 on the proliferation of OS cells, flow cytometry was conducted; the results demonstrated that knockdown FOXD2-AS1 expression may inhibit cell proliferation by preventing entry into the $\mathrm{S}$ phase of the cell cycle. The tumor model in nude mice revealed that downregulation of FOXD2-AS1 significantly slowed the growth of OS and reduced the potential for lung metastasis. These results indicated that FOXD2-AS1 may be a tumor-promoting factor.

To further explore the molecular mechanism by which FOXD2-AS1 exerts its effects on OS cells, the present study verified the localization of FOXD2-AS1 in the cytoplasm using FISH and RNA isolation experiments. In addition, the effects of FOXD2-AS1 knockdown on putative downstream targets were determined using RT-qPCR and western blotting. Alterations in the protein expression levels of PHGDH and RRM2 were detected in response to FOXD2-AS1 knockdown, thus suggesting that FOXD2-AS1 may regulate the expression of PHGDH and RRM2.

Abnormal expression of PHGDH and RRM2 serves a crucial role in various cancer behaviors, including tumor cell proliferation, apoptosis, drug resistance and metastasis $(28,29)$. It has been reported that high expression of RRM2 has an adverse effect on the survival and prognosis of patients with OS (30). Previous studies reported that lncRNAs act as ceRNAs and competitively bind microRNAs, in order to regulate mRNA expression; the corresponding microRNA 
A

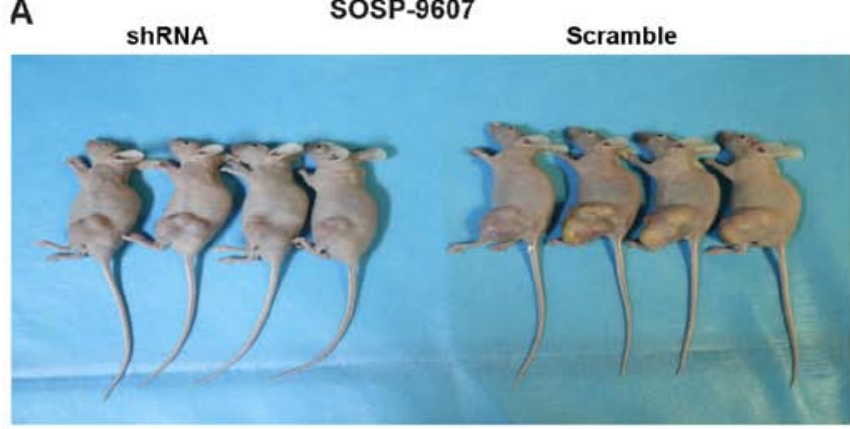

C

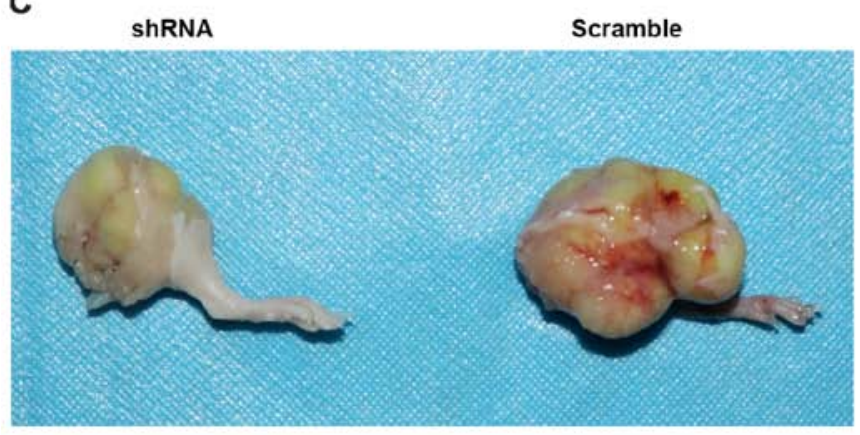

D

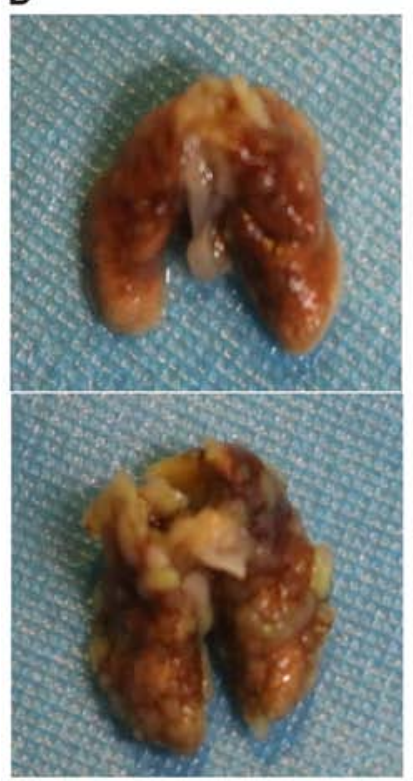

$\mathbf{F}$

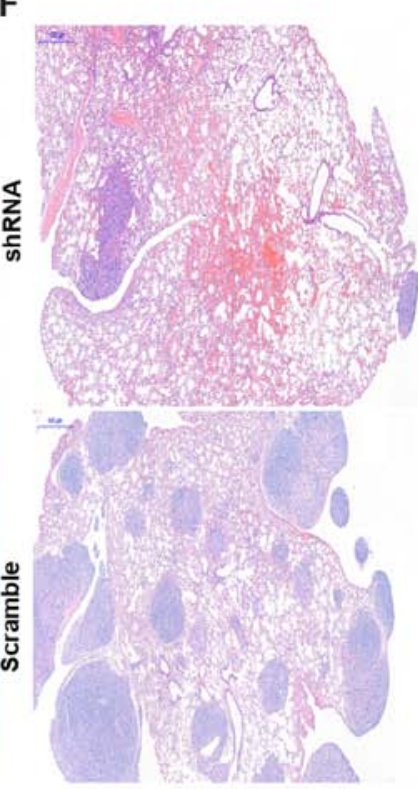

B

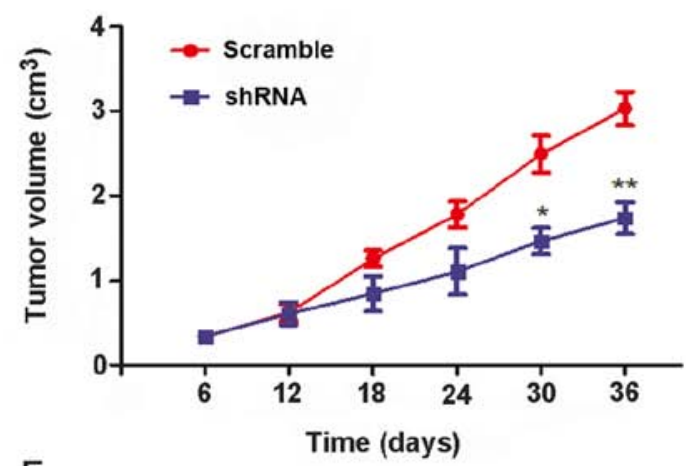

E

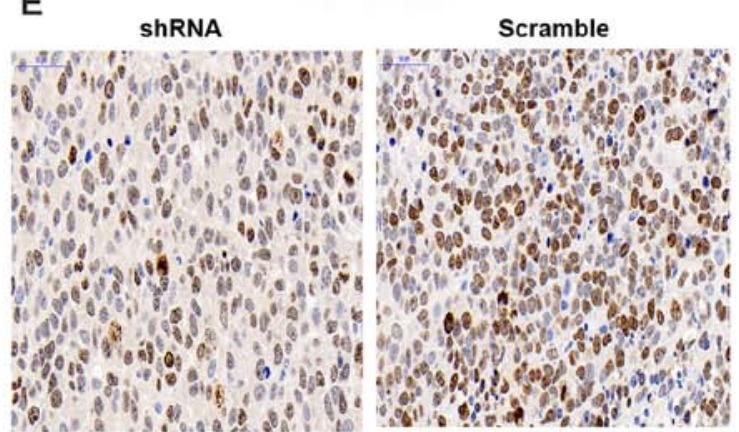

G

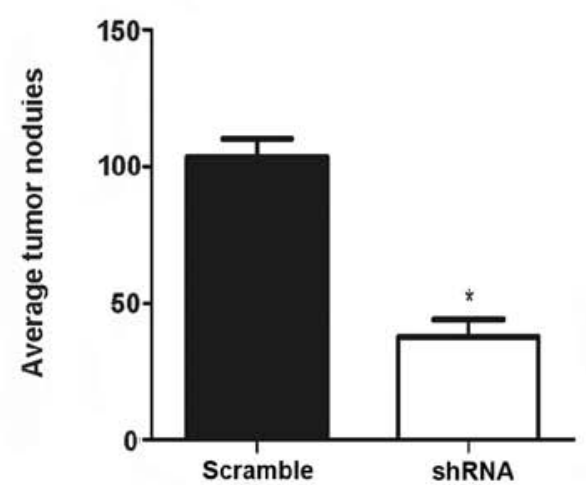

Figure 5. Knockdown of FOXD2-AS1 inhibits OS growth and lung metastasis in vivo. (A) Images of tumors derived from SOSP-9607 cells 36 days post-injection. (B) Growth curves of SOSP-9607 cells with stable expression of FOXD2-AS1 shRNA or scramble shRNA. (C and D) A total of 36 days post-inoculation with tumor cells, representative images of in situ tumors and lung metastases were captured. (E) Immunohistochemistry was performed on spontaneous orthotopic tumors in the experimental and scramble groups to detect the protein expression level of Ki-67. Magnification, $x 400$. (F) Hematoxylin and eosin staining of spontaneous lung metastases in the experimental and scramble groups. (G) Total number of lung nodules in the two groups. Representative images are shown in $(\mathrm{C}-\mathrm{F}) .{ }^{*} \mathrm{P}<0.05$ and ${ }^{* *} \mathrm{P}<0.01$ vs. scramble shRNA. Data are presented as the means \pm standard deviation. FOXD2-AS1, forkhead box D2 adjacent opposite strand RNA1; shRNA, short hairpin RNA.

response element is the basis of this interaction $(31,32)$. However, to the best of our knowledge, which microRNA FOXD2-AS1 binds as a ceRNA, in order to regulate the expression levels of PHGDH and RRM2, has not been studied. In addition, the mechanism by which FOXD2-AS1 regulates RRM2 and PHGDH has not been studied in the present study. Therefore, more experimental studies regarding the functional role of FOXD2-AS1 in OS are required.
In conclusion, the present findings demonstrated that FOXD2-AS1 was upregulated in OS cells and OS tissue samples compared with in normal osteoblasts and adjacent tissue samples. In addition, knockdown of FOXD2-AS1 expression inhibited OS cell proliferation, migration and invasion. However, the mechanism by which FOXD2-AS1 regulates the biological behavior of OS has not been investigated in this study. We aim to further investigate how FOXD2-AS1 regulates the 
expression of RRM2 and PHGDH in subsequent experiments. In conclusion, these findings suggested that FOXD2-AS1 may be a potential clinical therapeutic target, and further studies are required to elucidate the molecular mechanism underlying FOXD2-AS1-mediated OS progression.

\section{Acknowledgements}

Not applicable.

\section{Funding}

The present research was supported by research grants from the National Natural Science Foundation of China (grant nos. 81272441).

\section{Availability of data and materials}

The datasets used and/or analyzed during the current study are available from the corresponding author on reasonable request.

\section{Authors' contributions}

$\mathrm{HZ}$ and YL performed the functional in vitro assays. $\mathrm{HZ}, \mathrm{TZ}$ and $\mathrm{CD}$ performed qPCR and western blot assays. HZ, JW and XL performed the functional in vivo assays. $\mathrm{HZ}$ analyzed the data and wrote manuscript. XW collected and analyzed clinical samples. YZ, TY and QM designed the present study. All authors read and approved the final manuscript.

\section{Ethics approval and consent to participate}

The use of human OS tissue was approved by the Air Force Military Medical University Ethics Committee, and the collected specimens were obtained with the written informed consent of the patients or their families. The animal study was approved by the Animal Care and Use Committee of the Second Affiliated Hospital of the Air Force Medical University.

\section{Patient consent for publication}

Not applicable.

\section{Competing interests}

The authors declare that they have no competing interests.

\section{References}

1. Bishop MW, Janeway KA and Gorlick R: Future directions in the treatment of osteosarcoma. Curr Opin Pediatr 28: 26-33, 2016.

2. Yang Z, Li X, Yang Y, He Z, Qu X and Zhang Y: Long noncoding RNAs in the progression, metastasis, and prognosis of osteosarcoma. Cell Death Dis 7: e2389, 2016.

3. Lee $\mathrm{C}$ and Kikyo N: Strategies to identify long noncoding RNAs involved in gene regulation. Cell Biosci 2: 37, 2012.

4. Mattick JS: Non-coding RNAs: The architects of eukaryotic complexity. EMBO Rep 2: 986-991, 2001

5. Di Gesualdo F, Capaccioli S and Lulli M: A pathophysiological view of the long non-coding RNA world. Oncotarget 5 : 10976-10996, 2014.
6. Chang L, Li C, Lan T, Wu L, Yuan Y, Liu Q and Liu Z: Decreased expressionoflongnon-codingRNAGAS5indicatesapoorprognosis and promotes cell proliferation and invasion in hepatocellular carcinoma by regulating vimentin. Mol Med Rep 13: 1541-1550, 2016.

7. Lalevee S and Feil R: Long noncoding RNAs in human disease: Emerging mechanisms and therapeutic strategies. Epigenomics 7: 877-879, 2015.

8. Gutschner T and Diederichs S: The hallmarks of cancer: A long non-coding RNA point of view. RNA Biol 9: 703-719, 2012.

9. Smolle MA and Pichler M: The role of long non-coding RNAs in osteosarcoma. Noncoding RNA 4: pii: E7, 2018.

10. Su F, He W, Chen C, Liu M, Liu H, Xue F, Bi J, Xu D, Zhao Y, Huang $\mathrm{J}$, et al: The long non-coding RNA FOXD2-AS1 promotes bladder cancer progression and recurrence through a positive feedback loop with Akt and E2F1. Cell Death Dis 9: 233, 2018.

11. Xiong Y, Wang T, Wang M, Zhao J, Li X, Zhang Z, Zhou Y, Liu J, Jia L and Han Y: Long non-coding RNAs function as novel predictors and targets of non-small cell lung cancer: A systematic review and meta-analysis. Oncotarget 9: 11377-11386, 2018.

12. Zhang J, Fan D, Jian Z, Chen GG and Lai PB: Cancer specific long noncoding RNAs show differential expression patterns and competing endogenous RNA potential in hepatocellular carcinoma. PLoS One 10: e141042, 2015.

13. Chen X, Yang TT, Wang W, Sun HH, Ma BA, Li CX, Ma Q, Yu Z and Fan QY: Establishment and characterization of human osteosarcoma cell lines with different pulmonary metastatic potentials. Cytotechnology 61: 37-44, 2009.

14. Livak KJ and Schmittgen TD: Analysis of relative gene expression data using real-time quantitative PCR and the 2(-Delta Delta C(T)) method. Methods 25: 402-408, 2001.

15. Benjamin RS: Osteosarcoma: Better treatment through better trial design. Lancet Oncol 16: 12-13, 2015.

16. Durnali A, Alkis N, Cangur S, Yukruk FA, Inal A, Tokluoglu S, Seker MM, Bal O, Akman T, Inanc M, et al: Prognostic factors for teenage and adult patients with high-grade osteosarcoma: An analysis of 240 patients. Med Oncol 30: 624, 2013.

17. Miller BJ, Cram P, Lynch CF and Buckwalter JA: Risk factors for metastatic disease at presentation with osteosarcoma: An analysis of the SEER database. J Bone Joint Surg Am 95: e89, 2013.

18. Chan JJ and Tay Y: Noncoding RNA: RNA regulatory networks in cancer. Int J Mol Sci 19: pii: E1310, 2018.

19. Wang YL, Shao J, Wu X,Li T, Xu M and Shi D: A long non-coding RNA signature for predicting survival in patients with colorectal cancer. Oncotarget 9: 21687-21695, 2017.

20. Tuo YL, Li XM and Luo J: Long noncoding RNA UCA1 modulates breast cancer cell growth and apoptosis through decreasing tumor suppressive miR-143. Eur Rev Med Pharmacol Sci 19: 3403-3411, 2015.

21. Nie W, Ge HJ, Yang XQ, Sun X, Huang H, Tao X, Chen WS and Li B: LncRNA-UCA1 exerts oncogenic functions in non-small cell lung cancer by targeting miR-193a-3p. Cancer Lett 371: 99-106, 2016.

22. Arab K, Park YJ, Lindroth AM, Schäfer A, Oakes C, Weichenhan D, Lukanova A, Lundin E, Risch A, Meister M, et al: Long noncoding RNA TARID directs demethylation and activation of the tumor suppressor TCF21 via GADD45A. Mol Cell 55: 604-614, 2014.

23. Chen F, Mo J and Zhang L: Long noncoding RNA BCAR4 promotes osteosarcoma progression through activating GLI2-dependent gene transcription. Tumour Biol 37: 13403-13412, 2016.

24. Sun XH, Yang LB, Geng XL, Wang R and Zhang ZC: Increased expression of lncRNA HULC indicates a poor prognosis and promotes cell metastasis in osteosarcoma. Int J Clin Exp Pathol 8: 2994-3000, 2015.

25. Cao L, Wang Y, Wang Q and Huang J: LncRNA FOXD2-AS1 regulates chondrocyte proliferation in osteoarthritis by acting as a sponge of miR-206 to modulate CCND1 expression. Biomed Pharmacother 106: 1220-1226, 2018.

26. Yang X, Duan B and Zhou X: Long non-coding RNA FOXD2-AS1 functions as a tumor promoter in colorectal cancer by regulating EMT and Notch signaling pathway. Eur Rev Med Pharmacol Sci 21: 3586-3591, 2017.

27. Hainaut $P$ and Plymoth A: Targeting the hallmarks of cancer: Towards a rational approach to next-generation cancer therapy. Curr Opin Oncol 25: 50-51, 2013. 
28. Ou Y, Wang SJ, Jiang L, Zheng B and Gu W: p53 Protein-mediated regulation of phosphoglycerate dehydrogenase (PHGDH) is crucial for the apoptotic response upon serine starvation. J Biol Chem 290: 457-466, 2015.

29. Jing Z, Heng W, Xia L, Ning W, Yafei Q, Yao Z and Shulan Z: Downregulation of phosphoglycerate dehydrogenase inhibits proliferation and enhances cisplatin sensitivity in cervical adenocarcinoma cells by regulating Bcl-2 and caspase-3. Cancer Biol Ther 16: 541-548, 2015.

30. Fellenberg J, Bernd L, Delling G, Witte D and ZahltenHinguranage A: Prognostic significance of drug-regulated genes in high-grade osteosarcoma. Mod Pathol 20: 1085-1094, 2007.
31. Tay Y, Kats L, Salmena L, Weiss D, Tan SM, Ala U, Karreth F, Poliseno L, Provero P, Di Cunto F, et al: Coding-independent regulation of the tumorsuppressorPTEN by competing endogenous mRNAs. Cell 147: 344-357, 2011.

32. Salmena L, Poliseno L, Tay Y, Kats L and Pandolfi PP: A ceRNA hypothesis: The Rosetta Stone of a hidden RNA language. Cell 146: 353-358, 2011.

(c) (i) (9) This work is licensed under a Creative Commons Attribution-NonCommercial-NoDerivatives 4.0 International (CC BY-NC-ND 4.0) License. 